\title{
Study on the Engineering Property of Fiberglass-Polyester Paving Mat
}

\author{
Yi Chaojue and Zhai Pengcheng
}

\begin{abstract}
New compound synthetic material as Fiberglass-Polyester Paving Mat has been, it is a compound of fiberglass and polyester. Fiberglass-Polyester Paving Mat has a feature of high strength, good compatibility, low elongation, resistance of high temperature and corrosiveness. This paper mainly elaborated the performance of Fiberglass-Polyester Paving Mat, such as delaying cracking, alleviating the crack development, which can effectively prevent the road surface water infiltration and improve the pavement performance in asphalt pavement construction and transformation. Based on the forming mechanism of reflective cracks, this article describes the engineering application of fiberglass-polyester paving mat in the prevention of reflection crack of bituminous pavement and in waterproofing of the paving layer in tunnels, thus providing the basis for the application of Fiberglass-Polyester Paving Mat in highway construction and maintenance engineering.
\end{abstract}

Index Terms-Fiberglass-polyester paving mat, geotechnical synthetic material, reflective cracks, waterproofing crack resistance.

\section{INTRODUCTION}

Fiberglass-Polyester Paving Mat is a new glass fiber composite anti-cracking material, which is composed of $60 \%$ glass fiber and $40 \%$ polyester fiber comprising a composite geotechnical synthetic material. This unique combination provides the strength of glass fiber and the flexibility of polyester fiber without shortcomings of raw materials. Fiberglass-Polyester Paving Mat is following the geotextile, geogrid, strip polymer and later develop into a new type of material, meanwhile it has good compatibility with the asphalt mixture. Fiberglass-Polyester Paving Mat in road engineering generally arranged in the layer and the base layer of asphalt or between asphalt overlay and the old road, which, however, to improve reflection crack resistance capacity and fatigue life of asphalt concrete [1,2].

\section{ENGINEERING CHARACTER OF GEOTECHNICAL SYNTHETIC MATERIAL}

There is variety of geotechnical synthetic material, which was early divided into two categories called geotextiles and geomembrane, respectively representing the pervious and

Manuscript received on March 22, 2012; revised March 24, 2012. This work was supported in part by the Southwest Jiaotong University.

C. $\mathrm{Yi}$ is with the Southwest Jiaotong University, majored in structural engineering. (e-mail: wangluotianzi012@ sina.com).

P. Zhai is with the Southwest Jiaotong University, majored in railway engineering. (e-mail: 442672249@qq.com). impervious synthetic material. In recent years large scene of synthetic materials with polymer as raw material have appeared, composite materials, special materials products emerged largely, which exceeded the fabric and film category. Geotechnical synthetic material is now laying in many fields of engineering application because of its role in the following:

\section{A. Drainage Effect}

Some engineering synthetic materials such as fabric is porous permeable medium, buried in the upper or middle can congregate water, and then drain from the soil. These materials are now widely used in earth dam, embankment, retaining wall and soft foundation consolidation.

\section{B. Isolation Effect}

In geotechnical engineering, the different pellet layer occurs frequently among intermixing phenomenon, leading that each layer lost the due performance. The paving of geotechnical synthetic materials of different granular layer in between can isolate, drain and prevent the soil bearing capacity deterioration effect. The isolation effect of geotechnical synthetic materials in the soft foundation of highway, railway has good effects, by which can effectively prevent the interlayer material on the mutual penetration and control uneven settlement.

\section{Protection Effect}

On the use of the integrity of engineering synthetic materials, it can spread more concentrated stress; prevent structure damage under the action of external force, thus playing a protective role. Such protective material is mainly applied in the river bottom protection, revetment and coast moisture-proof; however, in road slope protection engineering, protective material is used to prevent the flow of current scour erosion and hypothermia caused by frost heave.

\section{Anti-Seepage Effect}

By using the uniformity, imperfection of geotechnical synthetic material, we set it into different structural layers in order to let the structure function gain seepage control.

\section{E. Filtering Effect}

In order to prevent the soil particle piping phenomenon, we generally use the graded aggregate for filtering layer, and a spinning or nonwoven fabrics can both replace the conventional granular filtering layer, which conduct the filter. But when the requirements for filtering layer are not very high, we generally make filter layer of nonwoven fabrics.

\section{F. Reinforcement Effect}

Some synthetic materials are of high tensile strength and 
good overall performance, if be set as reinforced material in a proper way into the engineering structure, they can deliver external load to the adjacent structure layer, so as to limit structure deformation, improve or enhance certain structure stability and improve the bearing capacity of structure.

\section{Project Characteristics OF Fiberglass-Polyester PAVING MAT}

In numerous geosynthetic materials, the Fiberglass-Polyester Paving Mat and Asphalt mix a whole, and become a waterproof layer with good compatibility. Their mixture can not only physically isolate moisture, but also has a better ductility. So the Fiberglass-Polyester Paving Mat is widely used.

Engineering practice [3], [4] shows that Fiberglass cannot resist water and it is hard to be fixed while easy to expand. When the Paving temperature of the mixture of Geotextile and hot Asphalt exceed $160{ }^{\circ} \mathrm{C}$, its intensity and Creep resistance is not enough good to play reinforcement effect. The Fiberglass-Polyester Paving Mat solves this problem between them. It can effectively maintain the crack and water damage on bridgedeck and pavement. Compared with other geotextiles, Fiberglass-Polyester Paving Mat has characteristics as following:

(1)Fiberglass-Polyester Paving Mat has low elongation without long-term creep degeneration, which guarantees manufacture can be used for a long time.

(2)Fiberglass-Polyester Paving Mat has compatibility and good high-temperature stability. Fiberglass-Polyester Paving Mat can be compatible with Asphalt and Asphalt mixture. Its melting point is over $230^{\circ} \mathrm{C}$ when it is both physically and chemically stable. So, under the hot asphaltic concrete of $170^{\circ} \mathrm{C}$, Fiberglass-Polyester Paving Mat would not be affected.

(3)Fiberglass-Polyester Paving Mat has chemical stability. After specially processed, Fiberglass-Polyester Paving Mat can avoid various chemical erosions.

(4)Fiberglass-Polyester Paving Mat combines with the hot asphalt a tacky layer, which builds a waterproof layer. Even if the surface could crack, it also can prevent the infiltration of water on the surface in effect in order to avoid the water damage to the base course.

(5)Fiberglass-Polyester Paving Mat can be smashed and recycle. That means it can be smashed into very small fiber to enhance the recycling material (RAP) in the milling drum.

In addition, Indifferent stress level the Fiberglass-Polyester Paving Mat can prolong asphalt mixture's life. The common fiberglass mat may improve the fatigue performance of asphalt mixture at the low stress level, while shorten its life at a high stress level.

IV. The Utilization of Fiberglass-Polyester PaVing MAT ON THE ASPECT OF PREVENTING REFLECTIVE CRACKS ON PAVEMENT

\section{A. The Causes of Reflective Cracks' Formation}

In a long-term time of using, semi-rigid base asphalt pavement will form Reflective Cracks more or less for the vehicles load recapitulate impaction and continual natural inflection. For instance, some cracks like cross joint and shaped crack are the most common damages of asphalt pavement. Many factors can lead to them, such as the low-quality base course which is the major reason to cause reflective cracks. If we do not repair the road, rain and other pollutants will infiltrate along the cracks into subgrade, which can decrease the bearing capacity, split up the cracks, damage the structure of pavement. This would influence road capacity and short the service life of pavement.

The causes of semi-rigid base asphalt road may involve three aspects as following: First, the surface itself is bad; Second, base course cracks for dry shrinkage and thermal shrinkage and it reflect on the surface; third, the surface and base course has reciprocities.

For semi-rigid base, cracks often are not caused by traffic load. The crushed stone cement base can easily form cracks for moisture evaporation and temperature changes. Before bearing the load, there have been a lot of small cracks. So, if fact, it bears traffic load with cracks existing and the dry shrinkage and thermal shrinkage of base course are the internal causes. The cause of Reflective Cracks is because the tensile stress at the bottom of surface exceeds the limit of asphalt concrete. When the base course cracks, it loses the function of resisting tensile stress. Then it passes the tensile stress to the surface at the crack and leads the tensile stress collection on the surface. If there were a shearing stress at the same time, the tensile stress would exceed the limit of material. Then, the surface cracks. The shearing stress of off-axis load helps to cause Reflective Cracks. Therefore, Reflective Cracks are mainly caused by horizontal and vertical displacement of the base course. Mechanism of reflection cracks in Figure 1 and Figure 2.

\begin{tabular}{|c|c|}
\hline Asphalt & \ Crack \\
\hline Cement Stabilized & $\overrightarrow{\text { Temperature }}$ \\
\hline
\end{tabular}

Fig. 1.Temperature contraction induced by horizontal tensile stress of the reflective cracks.

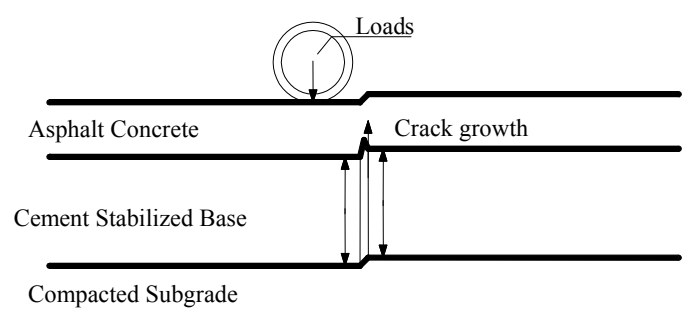

Fig. 2. Traffic load induced by vertical tensile stress of the reflection cracks.

\section{B. The function of the Fiberglass-Polyester Paving Mat in the reducing of Grassroots crack}

To prevent and mitigate the reflection cracks to extend the maintenance cycle of the road, in between the road base and surface layer with a layer of geotextile fabric, the surface layer and the inter-layer between the grass-roots level, can play the role of crack gentle. By the preceding bout of the 
polyester fiberglass know, stress cracks on the grassroots level through horizontal polyester fiberglass cloth can be extended to a wider range of stress, thus easing the stress concentration at the crack, absorb part of the stretch play the role of energy, improve stress distribution pavement structure to resist and delay caused by the asphalt base crack because the occurrence of reflective cracking.

Polyester fiberglass cloth saturated with asphalt to form impermeable layer of viscous oil, can prevent the infiltration of surface water, thereby reducing the primary softening reduce flooding reflective cracking in the asphalt surface layer deboning at the crack surface water infiltration along, vehicles moving at high loads, high-speed movement of water and the surface layer of the primary dangers are great addition, thermal insulation, reducing the surface temperature gradient.

Furthermore, polyester fiberglass cloth surface layer and reduced the binding force between the grassroots, so that the surface layer of the maximum tensile strain is also reduced. To further study the polyester fiberglass cloth to reduce the impact of primary cracks, do the following finite element simulation:

As the experimental conditions, this study analyzed only the type of reflective cracking load and the design of the indoor simulation model.

Paper, only semi-rigid base asphalt pavement has cracked the case, head cracks throughout the primary and sub-base, have a certain thickness, without considering the combined effects of temperature stress, according to previous studies [5], and the inverse of the reference test road modulus, surface structure calculated using the model.

\begin{tabular}{|c|c|c|}
\hline Asphalt & $\mathrm{h}=20 \mathrm{c}$ & $\mathrm{E}=1400 \mathrm{MPa}$ \\
\hline Cement Stabilized & $\mathrm{h}=30 \mathrm{c}$ & $\mathrm{E}=3500 \mathrm{MPa}$ \\
\hline Compacted & $\mathrm{h}=35 \mathrm{c}$ & $\mathrm{E}=600 \mathrm{MPa}$ \\
\hline Natural & $\mathrm{h}$ ? & $\mathrm{E}=60 \mathrm{MPa}$ \\
\hline
\end{tabular}

Fig. 3. Semi-rigid base has been cracked finite element simulation.

TABLE I: STATISTICAL TABLE OF THE FINITE ELEMENT ANALYSIS RESULTS

\begin{tabular}{|c|c|c|c|}
\hline & $\begin{array}{l}\text { Ordinary } \\
\text { asphalt } \\
\text { concrete } \\
\text { material }\end{array}$ & $\begin{array}{c}\text { Fiberglass-Polyester } \\
\text { Paving Mat } \\
(E=300 \mathrm{MPa})\end{array}$ & $\begin{array}{c}\text { Fiberglass-Polyes } \\
\text { ter Paving Mat } \\
(\mathrm{E}=1000 \mathrm{MPa})\end{array}$ \\
\hline $\begin{array}{c}\text { The } \\
\text { max-tens } \\
\text { ile stress } \\
\text { of crack } \\
\text { tip }\end{array}$ & $0.69 \mathrm{MPa}$ & $0.63 \mathrm{MPa}$ & $0.53 \mathrm{MPa}$ \\
\hline
\end{tabular}

PS: The crack width $1 \mathrm{~cm}$.

Experiments with ordinary asphalt surface mixture, set between the surface layer and the primary materials of polyester fiberglass cloth laminated. Layers by assuming homogeneous, isotropic elastic material composition; layers are infinite in the horizontal direction; layers are completely continuous self-respect does not count the impact of the pavement structure.

From the above results and process knowledge, (1) plus geosynthetic tensile stress after the crack tip significantly reduced, indicating that geosynthetics can delay the spread of reflective cracking; (2) with geosynthetics to increase the elastic modulus, fracture tip of the tensile stress significantly reduced, indicating that geosynthetics can delay the spread of reflective cracking.

\section{The APPliance of FiBerglass-Polyester PAVING} MAT IN WATERPROOFING OF THE PAVING LAYER IN TUNNELS

Usually, there would be waterproof layer positioned on the surface of the joint of the cement concrete planks in the road tunnels, then, attached by the upper and middle layers which were constructed the same as the road bases. The crevices and the inappropriate joint would lower the stability and the entirety of the whole construction which will cause unequal stress on different layers. In the occasions of the drainage in the tunnels is not utilized or when the underground water goes up, the water will permeate into the asphalt layer, these phenomena would enlarge the possibility of road early damages to occur.

Right now, the most frequent used waterproofing and cracks resistant method is a composed one: using the SBS modified asphalt and waterproof coiled materials to tackle with the joint of the cement concrete planks, or over covering fiber-glass grids and applying waterproofing paint, the entire measure is depended on the physical waterproofing functions of the materials themselves. But, there are many shortages dealing with the problems this way, the waterproofing paint is easy to be damaged or become aged when painting, after painted, and in the following asphalt paving period, those damages would have severe negative impact on the waterproofing effect of the painting material itself [6].

Depending on its characteristics, the Fiberglass-Polyester Paving Mat has some positive effect on waterproofing and crack resistance. There is something need to be noticed that the adhesive property between different layers is pivotal to the function of the Fiberglass-Polyester Paving Mat. If the layer were not well adhered after the mat is paved, the shear stress brought by the vehicles when speeding up or breaking would probably segregate the layer whereas the mat is useless. Furthermore, coping with the problem of the underground water and compared with some other material or results in some experiments [7], the Fiberglass-Polyester Paving Mat appeared to be more functional when soaked in water and attenuated slightly.

\section{Application of Fiberglass Polyester PaVing Mat IN WATERPROOF COHESIVE LAYER ON DECK}

As previously mentioned, Fiberglass Polyester Paving Mat is a new type of composite geotechnical synthetic material, and has good compatibility with asphalt mixture, thus combined into a waterproof layer. Putting Fiberglass Polyester Paving Mat into the bridge deck waterproof adhesive layer to make full use of the characteristics of high strength and high toughness, making Fiberglass Polyester Paving Mat and asphalt stress absorbing membrane of viscosity reservoir formation, in overlay with asphalt or 
cement concrete plate or between pavement and formed between the buffer layer, to avoid stress concentration to reduce or inhibit reflection crack. In the application process, we mainly focus on the problems that whether the interlayer slip and adhesive force of interlayer.

In general coiled materials or coating materials chosen for waterproof adhesive layer in pavement of bridge deck on highways do not exhibit optimal effect. In order to improve the adhesive property between deck and asphalt pavement, the modified asphalt + fiberglass polyester paving mat are selected as waterproof adhesive materials. The dosage of oil in adhesive layer is determined via shearing test, the test road is paved and the pull test is carried out.

Research shows that, in the premise of reasonable construction, Bridge deck asphalt pavement for the waterproof and cohesive layer shear perform optimally, SBS modified bitumen in the optimum dosage of $1 \sim 1.2 \mathrm{~L} / \mathrm{m} 2$. This leads to good adhesion and penetration resistance of asphalt mixture. This is because when the adhesion layer of oil spraying quantity is less, the interlayer viscous effects is small, so the pavement interlayer shear capacity is little improved. but Along with the adhesive layer of oil spraying volume continues to increase, interlayer cohesive function increases ceaselessly, but if the sticky layer of oil spraying quantity is overmuch, layer between the thin film layer will thicken, which greatly weakened the pavement below two mutually interlocking and friction, so that the structure of interlayer shear capacity reduce.

\section{CONCLUSION}

Through using Fiberglass-Polyester Paving Mat, the tensile stress and vertical shearing stress of bitumen's bottom can be decreased. With the condition of satisfying structural bearing capacity, using Fiberglass-Polyester Paving Mat can, to some degree, make bitumen thinner.

The tensile stress of bitumen's bottom is weaker if the integrity with upper or lower layer is strong due to high bond property from anti-cracking material. The function of anti-crack of those materials could be blocked with weak bond property, which will cause the slide of interface layers and pushing destroy of bitumen.

According to some researches, the property of bond with upper or lower level of the anti-crack material is the main determinant of the effect of anti-crack. Thus, it is critical to choose the anti-crack material with extremely good initial bond property, making sure its function could be lasted longer during repetitive everyday use.

Fiberglass-Polyester Paving Mat is a new compound synthetic material, and a compound of fiberglass and polyester, which contain the flexibility of polyester fiber and intensity of glass fiber. Its advantages include high strength, low elongation, good compatibility with bitumen, resistance of high temperature and corrosiveness. It can be used for construction and reconstruction of bitumen pavement to prevent reflective crack and water damage and to strengthen the bearing capacity of the pavement, with fair application values in the practical projects.

\section{REFERENCES}

[1] Z. Zhou and X. Ming, "Fiberglass-Polyester Paving Mat and asphalt mixture performance test," Technology of highway and transport, no.7, 2010.

[2] Highway construction technology of modified asphalt pavement specification(JTGF 40-2004), China Communications Press, JTGF 40-2004

[3] C. Y. Lin, M. Wu, J. A. Bloom, I. J. Cox, and M. Miller, "Rotation, scale, and translation resilient public watermarking for images," IEEE Trans. Image Process., vol. 10, no. 5, pp. 767-782, May 2001.

[4] X. Wang, Z. Wang, "Geotechnical synthetic materials for prevention of reflection crack of bituminous pavement design," China highway, no. 6, 2003.

[5] D.. R. Jones IV, "TruPave Testing Methods- Super Pave IDT at Univ. of Florida," August 3,2003,USA

[6] M. Li and X. Wang, "Adhesion between asphalt pavement layers on water damage analysis," Technology of highway and transport, no.3, 2008.

[7] H. When, N. Wu, and H., Zhang, "The comparative research on effect of Fiberglass-Polyester Paving Mat and glass fiber grid to prevent cracks in cement stabilized macadam base," Technology of highway and transport, no. 5, 2010. 